\title{
SUSY GUTs with Yukawa Unification in the light of data
}

\section{Diego Guadagnoli *}

Physik-Department, Technische Universität München, James-Franck-Straße, 85748 Garching,

Germany

Excellence Cluster “Universe”, Boltzmannstraße 2, D-85748 Garching, Germany

E-mail: diego.guadagnoli@ph.tum.de

I provide a short overview of the hypothesis of Yukawa unification within SUSY GUTs, emphasizing its motivations and its predictivity-enhancing role. I then discuss the status of the tests of this hypothesis, and the major role played by observables in the flavor sector. Concerning the patterns of soft SUSY-breaking terms assumed at the GUT scale, I focus on the cases of universalities on sfermion and gaugino masses, as well as of non-universalities of minimally flavor-violating type, hence inheriting from the unified Yukawa coupling.

European Physical Society Europhysics Conference on High Energy Physics, EPS-HEP 2009, July 16 - 222009

Krakow, Poland

${ }^{*}$ Work supported by the A. von Humboldt Stiftung and in part by the Cluster of Excellence "Origin and Structure of the Universe". 


\section{Yukawa Unification}

It remains a striking fact that the Standard Model (SM) gauge couplings, measured at low energies, evolve to a single value at a high scale $M_{G} \approx 3 \times 10^{16} \mathrm{GeV}$, provided that, above the electroweak scale, the SM is assumed to become supersymmetric. Barring a coincidence - that a more in-depth look at this coupling unification does not actually support - this fact hints at the existence of a larger, simple gauge group embedding the SM one above $M_{G}$, the corresponding theory being dubbed a Grand Unified Theory (GUT). The cornerstone predictions of supersymmetric GUTs are $^{1}$, besides (i.) gauge coupling unification at $M_{G}$, (ii.) the existence of Supersymmetry (SUSY) throughout the energy interval between $M_{G}$ and $M_{\mathrm{EW}}$ (hence TeV-scale sparticles), and (iii.) proton decay. While the occurrence of $(i$.) can be assessed fairly model-independently within the Minimal Supersymmetric Standard Model (MSSM), precise predictions for (ii.) and (iii.) depend in general on a body of model assumptions. In particular, prediction (ii.) maps onto the question of the unknown mechanism of SUSY breaking and of the form that Yukawa couplings assume at the high scale. A very elegant assumption, in this respect, is that of GUT-scale Yukawa Unification (YU). Since this unification can in general be spoiled by e.g. the presence of higher-dimensional interactions, the crucial question is whether YU may leave any low-energy remnant at all. While for the light fermion generations this is definitely not the case, for the $3^{\text {rd }}$ generation it is an open and appealing possibility, selecting the group $\mathrm{SO}(10)$ as the most predictive case. In this case, verification of the YU hypothesis would amount to the important conclusion that all the dimension-4 interactions involving 3rd-generation fermions and/or scalars originate from just the $\mathbf{1 6}_{\mathbf{3}} \mathbf{1 6}_{\mathbf{3}} \mathbf{1 0}_{\mathbf{H}}$ structure. More shaky are symmetry assumptions, e.g. universality, on the Lagrangian terms associated with the breaking of SUSY, which correspond to operators of dimension less than 4, and are hence unlikely to preserve information about the symmetries inherent to the UV theory completion. The tacit motivation here is just one of computational simplicity.

\section{Testing Yukawa Unification}

YU is therefore a well-motivated, predictivity-enhancing hypothesis for SUSY GUTs. How to test it? The hypothesis of YU gives rise to two relations between the top quark, bottom quark and tau lepton masses. These relations depend on the renormalization group (RG) evolution, which is governed by gauge and Yukawa couplings only, and on weak scale threshold corrections, which depend on the soft SUSY-breaking parameters, or, equivalently, on the sparticle spectrum and mixings. These threshold corrections are most important for the $b$ quark due to non-holomorphic contributions enhanced by $\tan \beta$ [2]. Neglecting these threshold corrections, and requiring the tau and top masses to reproduce the observed values, the running $b$ quark mass $m_{b}\left(m_{b}\right)$ would be predicted to be $4.5 \mathrm{GeV}$, as opposed to the precisely measured experimental figure of (4.20 \pm 0.07$) \mathrm{GeV}$ [1]. This illustrates the necessity of considering regions of SUSY parameter space where the overall threshold corrections to $m_{b}$ are negative. An effective strategy to address this problem is to use the measured masses and the requirement of YU to see whether a special region in the theory parameter space emerges [3]. In fact it does. Under the simplifying assumption of universal GUT-scale soft terms for sfermions, $m_{16}$, and for gauginos, $m_{1 / 2}$, and with a positive $\mu$ parameter, YU prefers

\footnotetext{
${ }^{1}$ See S. Raby, Review on "Grand Unified Theories", in [1].
} 
the region characterized by $[3,4]^{2}$

$$
-A_{0} \approx 2 m_{16}, \mu, m_{1 / 2} \ll m_{16},
$$

where low-scale threshold corrections to $m_{b}$ are just the right -some $\%$ needed.

However, relations (2.1), together with the large value of $\tan \beta \approx 50$ required for $\mathrm{YU}$, have an important impact on the SUSY spectrum and on the predictions for flavor-changing neutral currents (FCNCs), in particular on those $B$-physics decay modes that are especially sensitive to large $\tan \beta$ and to the large trilinear coupling $A_{t}$ implied by relations (2.1). Specifically, the decay modes that turn out to have the strongest impact are $B_{s} \rightarrow \mu^{+} \mu^{-}, B \rightarrow X_{s} \gamma$ and $B \rightarrow X_{s} \ell^{+} \ell^{-}$. For example, in the parameter space (2.1), one typically has to face a substantial enhancement of $\operatorname{BR}\left(B_{s} \rightarrow \mu^{+} \mu^{-}\right)$ and huge destructive interference from chargino contributions in $\operatorname{BR}\left(B \rightarrow X_{s} \gamma\right)$.

In ref. [6] the non-trivial interplay among these observables and the bottom mass has been studied extensively through a fitting procedure. The main conclusions were:

(a) that a generic SUSY GUT with exact YU and GUT-scale universalities for sfermions and gauginos is phenomenologically viable only by advocating partial decoupling of the sfermion sector, the lightest mass exceeding $1 \mathrm{TeV}$;

(b) that phenomenological viability can be recovered without decoupling by relaxing $t-b-\tau$ unification to $b-\tau$ unification, equivalent to a parametric departure of $\tan \beta$ from the value implied by exact YU. This solution is non-trivial since, while the FCNC constraints prefer lower values of $\tan \beta$, a successful prediction of $m_{b}$ in YU requires instead a value of $\tan \beta$ very close to 50 [7]. Indeed, a compromise solution between the two classes of constraints has been found to exist only for the narrow range $46 \lesssim \tan \beta \lesssim 48$, implying that the breaking of $t-b \mathrm{YU}$ must be moderate, in the range $10-20 \%$.

It is worth mentioning that the parameter space in eq. (2.1) has been the topic of many other recent studies, with interesting differences in the approach and in the considered observables (see in particular [8]). For (some) comparisons I refer the reader to ref. [6].

\section{Beyond soft-terms universalities}

In ref. [9] it was subsequently considered the case of general SUSY GUT frameworks with YU enforced exactly, but where instead the hypothesis of universal GUT-scale soft terms (not very justified in itself) is relaxed. In particular, we entertained the general possibility that soft terms be of minimally flavor violating form [10]. In this case the requirement of exact YU and the hierarchical structure of the Yukawa couplings allow to parameterize squark bilinears and trilinears in a general, simple and accurate way [9]. Among the soft-term non-universalities allowed by this general parameterization, we have then focused on the scenario where up-type and down-type trilinear soft terms are split from each other.

\footnotetext{
${ }^{2}$ Quite interestingly the same relations emerge as fixed-point solution from the attempt to build SUSY models with radiatively-driven inverted scalar mass hierarchy (ISMH) [5], i.e. light third generation and heavy first and second generation sfermions. ISMH is an appealing possibility to relieve at one stroke the problem of fine tuning in the Higgs mass corrections, and of large flavor-changing neutral currents (FCNCs).
} 
This trilinear splitting scenario has been explored by contrasting the model predictions for EW observables, quark masses and quark FCNC processes against data in a global fitting procedure similar to that in ref. [6]. Agreement with data singles out one main scenario, featuring a sizable splitting between the $A$-terms and a large $\mu$-term $\approx m_{16}$. In spite of a slight increase in the fine tuning required to achieve EWSB with precisely the correct value of $M_{Z}$, this scenario allows a substantial improvement on other observables that, on a model-dependent basis, do often require some amount of fine tuning as well. First, and quite remarkably, phenomenological viability does not invoke a partial decoupling of the sparticle spectrum, as in the case of universal soft terms, but instead it requires part of the spectrum, notably the lightest stop, the gluino and the lightest chargino and neutralinos, to be very close to the current experimental limits. The lightest Higgs particle is also well above the LEP bound, it is actually quite robustly predicted at around $125 \mathrm{GeV}$. Second, the above parameter space is selected by a non-trivial interplay between the requirement of negative, sizable SUSY threshold corrections to $m_{b}$, and an instead negligible modification of the $B \rightarrow X_{s} \gamma$ decay rate, in presence of various other EW and $B$-physics constraints. Hence the very same mechanism that makes the $m_{b}$ correction large enough, automatically allows all FCNC constraints to be fulfilled. For all the details on the mechanism, see ref. [9].

Ref. [9] finally discussed a possible model of SUSY breaking where the pattern of soft terms, selected above on sheer phenomenological grounds, is realized. The results of ref. [9] provide a concrete example where, exploiting the full predictive power of YU and under what can be considered very plausible assumptions for soft terms, enough remnant information on the highenergy symmetries does indeed survive at low energies for these symmetries to be reconstructible.

\section{References}

[1] C. Amsler et al. [Particle Data Group], Phys. Lett. B 667 (2008) 1.

[2] L. J. Hall, R. Rattazzi and U. Sarid, Phys. Rev. D 50, 7048 (1994).

[3] T. Blazek, R. Dermisek and S. Raby, Phys. Rev. Lett. 88, 111804 (2002); T. Blazek, R. Dermisek and S. Raby, Phys. Rev. D 65, 115004 (2002).

[4] H. Baer and J. Ferrandis, Phys. Rev. Lett. 87, 211803 (2001).

[5] J. A. Bagger et al. Phys. Lett. B 473, 264 (2000).

[6] W. Altmannshofer, D. Guadagnoli, S. Raby and D. M. Straub, Phys. Lett. B 668 (2008) 385. See also: M. Albrecht, W. Altmannshofer, A. J. Buras, D. Guadagnoli and D. M. Straub, JHEP 0710 (2007) 055. D. Guadagnoli, arXiv:0810.0450 [hep-ph].

[7] M. S. Carena, S. Pokorski and C. E. M. Wagner, Nucl. Phys. B 406 (1993) 59.

[8] K. Tobe and J. D. Wells, Nucl. Phys. B 663, 123 (2003). D. Auto, H. Baer, C. Balazs, A. Belyaev, J. Ferrandis and X. Tata, JHEP 0306 (2003) 023. H. Baer, S. Kraml, S. Sekmen and H. Summy, JHEP 0803, 056 (2008). H. Baer, S. Kraml, S. Sekmen and H. Summy, JHEP 0810, 079 (2008). H. Baer, M. Haider, S. Kraml, S. Sekmen and H. Summy, JCAP 0902, 002 (2009). H. Baer, S. Kraml and S. Sekmen, arXiv:0908.0134 [hep-ph].

[9] D. Guadagnoli, S. Raby and D. M. Straub, arXiv:0907.4709 [hep-ph].

[10] G. D’Ambrosio, G. F. Giudice, G. Isidori and A. Strumia, Nucl. Phys. B 645, 155 (2002). G. Anderson et al., arXiv:hep-ph/9609457. 